\title{
OUTPUT-ONLY MODAL IDENTIFICATION OF TENSEGRITY STRUCTURES
}

\author{
Miniar ATTIG ${ }^{\mathrm{a}}$, Maher ABDELGHANI ${ }^{\mathrm{b}}$, Nabil ben KAHLA ${ }^{\mathrm{c}}$ \\ ${ }^{a}$ Ecole Polytechnique de Tunisie, University of Carthage, Applied Mechanics \\ and Systems Research Laboratory, Tunis, Tunisia \\ ${ }^{b}$ Higher Institute of Applied Sciences and Technologies of Sousse, University of Sousse, Sousse, Tunisia \\ ${ }^{c}$ King Khalid University, Civil Engineering Department, Abha, Saudi Arabia
}

Received 13 February 2016; accepted 04 April 1016

\begin{abstract}
Tensegrity systems are a special class of spatial reticulated structures that are composed of struts in compression and cables in tension. In this paper, the performance of stochastic subspace algorithms for modal identification of complex tensegrity systems is investigated. A sub-class algorithm of the Stochastic Subspace Identification family: the Balanced Realization Algorithm is investigated for modal identification of a tripod simplex structure and a Geiger dome. The presented algorithm is combined with a stabilization diagram with combined criteria (frequency, damping and mode shapes). It is shown that although the studied structures present closely spaced modes, the Balanced Realization Algorithm performs well and guarantees separation between closely-spaced natural frequencies. Modal identification results are validated through comparisons of the correlations (empirical vs. model based) showing effectiveness of the proposed methodology.
\end{abstract}

Keywords: system identification, stochastic subspace algorithm, balanced realization algorithm, tensegrity structures, Geiger dome, vibration.

\section{Introduction}

Tensegrity systems are a special class of spatial reticulated structures that are composed of struts and tendons. Tensioned and compressed components are assembled in a self-equilibrated system providing stability and stiffness to the structure. The word tensegrity comes from the contraction of tensile and integrity. It was proposed by Richard Buckminster Fuller in 1962. A recent and widely accepted definition was proposed by Motro (2003): "A tensegrity is a system in stable self-equilibrated state comprising a discontinuous set of compressed components inside a continuum of tensioned components". This definition includes systems where compressed elements are interconnected as tensegrity structures. Skelton et al. (2001) proposed the term "class $\mathrm{k}$ " to distinguish the different types of structures included in this broader definition. A "class $\mathrm{k}$ " tensegrity structure is defined as a stable tensegrity with a maximum of $\mathrm{k}$ interconnected compressive members.

The tensegrity concept has received significant interest among scientists and engineers in various fields ranging from aerospace, robotic, architecture and civil engineering to modelling of biological systems (Skelton, de Oliveira 2009). In aerospace technology, the tensegrity concept offers an alternative solution to design lightweight deployable structures as masts, solar arrays and antennas (Puig et al. 2010). The tensegrity paradigm has also inspired research on string-driven robotic systems (Graells Rovira, Mirats Tur 2009; Juan, 
Mirats Tur 2008; Paul et al. 2006). Architects are investigating responsive architecture and use of tensegrity systems in adaptive buildings (d'Estrée Sterk 2003). Furthermore, the tensegrity concept is used to model biological systems such as cytoskeleton structures of unicellular organisms (Ingber 1998). Tensegrities are also particularly attractive for active structures as often small amounts of energy are needed for structural control purposes and adaptation in changing environments (Skelton et al. 2001).

Research into dynamic behaviour of tensegrity structures was initiated in the mid-1980s.

Motro et al. (1986) presented experimental and numerical work on a tensegrity structure composed of three bars and 9 tendons. They showed that a linearized dynamic model around an equilibrium configuration offers a good approximation of the nonlinear behaviour of simple tensegrity structures. Ben Kahla et al. (2000) developed a numerical procedure for nonlinear dynamic analysis of tensegrity systems. Murakami (2001a, 2001b) used Lagrangian and Eulerian approaches to derive the equations of motion of tensegrity structures and performed numerical simulations and modal analysis of some tensegrity modules. Sultan et al. (2002) derived linearized dynamic models for two classes of tensegrity structures and showed that the modal dynamic range generally increases with the pretension. Arsenaultdub and Gosselin (2006) developed dynamic models of planar tensegrity modules with 1, 2 and 3 degrees of freedom. Masic and Skelton (2006) used a linearized dynamic model to enhance the dynamic control performance of a tensegrity structure. Dubé et al. (2008) presented a comparative study between experimental tests and numerical simulations carried out on a tensegrity minigrid considering static as well as dynamic loading. Recently, Bel Hadj Ali and Smith (2010) investigated dynamic behaviour of a five-module tensegrity structure and showed that the system resonant frequencies are closely related to the level of pretension. Korkmaz et al. (2011, 2012), have worked on determining control strategies for damage tolerance of an active tensegrity structure as well as on configuration of control system for deployment and damage tolerance of a tensegrity bridge. El Ouni and Ben Kahla (2014) studied the active vibration control of a Geiger dome using decentralized collocated integral force feedback.

Most studies on tensegrity dynamics showed that for such systems the structure modal signature is relat- ed to its state of self-stress. In fact, in tensegrity structures, the self-stress state significantly influences soft modes (zero-stiffness modes related to the structure infinitesimal mechanisms). This suggests that identifying the modal parameters and monitoring their possible changes may result in the evaluation of the system internal forces and the detection of possible damages of its components. However, the structure modal parameters are not easily identified especially when the natural frequencies are closely spaced which is generally the case for tensegrity structures (Bel Hadj Ali, Smith 2009). This arises the challenge of choosing the appropriate identification technique for such systems. The traditional identification techniques that extract modal parameters from input and output data have been well developed and widely used in engineering. However, it is often a hard task to carry out excitation in field testing for large structures. Moreover, in many cases, ambient excitation is of complex nature and cannot be measured (Off-shore structures, bridges, etc...). To obviate difficulties of traditional techniques, methods of extracting modal parameters from structural response data only have been deeply investigated during the past few decades (Mrabet et al. 2014; Reynders et al. 2007).

Several identification techniques such as Natural Excitation Technique (NEXT), Frequency Domain Decomposition (FDD) and Stochastic Subspace Identification (SSI) are employed to identify dynamic characteristics when structures are excited by unknown input. The basic assumption behind such techniques is that the excitation is a white Gaussian noise. Detailed knowledge of the excitation input is thus no longer need.

There has been much work on output-only identification. Benveniste and Fuchs (1985) considered the use of stochastic realization algorithms for structure modal analysis. Output-only system identification methods have been proven efficient for system identification in many engineering applications (Basseville et al. 2000). Among available techniques, the data driven Stochastic Subspace Identification techniques is considered to be one of the powerful identification techniques for output-only modal analysis in time domain (Giraldo et al. 2009). Van Overchee and De Moor (1996) provided a mathematical background for subspace algorithms existing in the literature, including those used to identify input-output systems or stochastic output-only systems. Several applications 
of output-only identification have been reported in the literature. Basseville et al. (2000) investigated subspace-based fault detection for vibration monitoring. Modal analysis of mechanical and civil structures is a classical and widely studied subject (Magalhaes et al. 2008; Carden, Mita 2009; Reynders et al. 2007). The output-only identification of complex tensegrity systems has not been studied.

In this paper, a sub-class algorithm of the SSI family: the Balanced Realization Algorithm is investigated for modal identification of a tripod simplex structure and a Geiger dome. The tensegrity structures are assumed to be time invariant in a taut and stiff configuration, experiencing small and linear oscillations about their stable equilibrium position, allowing the structures to be considered stationeries. The structures are symmetric and present closely spaced modes. The Balanced Realization Algorithm is presented and applied for output-only modal identification.

A stabilization diagram with high criteria (frequency, damping and mode shapes) is employed. Modal identification results are validated through comparisons of the correlations (empirical vs. model based) showing effectiveness of the proposed methodology.

\section{Dynamic model}

Let's assume that the behaviour of the studied structure can be described by a stationary linear dynamic system, and that, in the frequency range of interest, the input forces can be modelled as a stationary white noise. Consequently the model can be described through the following matrix differential equations:

$$
\left\{\begin{array}{l}
M \ddot{Z}(t)+C \dot{Z}(t)+K Z(t)=v(t) \\
Y(t)=L Z(t)
\end{array}\right.
$$

where $t$ denotes continuous time, $M, C$ and $K$ are the mass, damping and stiffness matrices respectively, (high dimensional) vector $Z$ collects the displacements of the degrees of freedom of the structure; the external (non measured) force $v$ is modelled as a stationary white noise with covariance matrix $Q$, measurements are collected in the (low dimensional) vector $Y$, and matrix $L$ states where the sensors are located (sensors positions). The mechanical characteristics ( $M, C$ and $K)$ of the system cannot be recovered from the output measurements. Hence, identifiable modal characteristics of the system are introduced: the vibration modes or eigen-frequencies denoted generically by $\mu$ and the modal shapes or observed eigenvectors denoted generically by $\psi_{\mu}$.

These quantities are solutions of the following equation:

$$
\left(M \mu^{2}+C \mu+K\right) \Psi_{\mu}=0, \quad \Psi_{\mu}=L \Psi_{\mu} .
$$

Sampling model represented by Equation (1) at rate $1 / \tau$ yields the discrete time model in state space form:

$$
\left\{\begin{array}{l}
X_{k+1}=F X_{k}+\varepsilon_{k} \\
Y_{k}=H X_{k}
\end{array}\right.
$$

where the state and the output are:

$$
X_{k}=\left[\begin{array}{c}
Z(k \tau) \\
\dot{Z}(k \tau)
\end{array}\right], Y_{k}=Y(k \tau)
$$

The state transition and observation matrices are:

$F=e^{\bar{F} T_{s}}, \bar{F}=\left[\begin{array}{cc}0 & I \\ -M^{-1} K & -M^{-1} C\end{array}\right], H=\left[\begin{array}{ll}L & 0\end{array}\right],(5 \mathrm{a}, \mathrm{b}, \mathrm{c})$

and where state noise $\varepsilon_{\mathrm{k}+1}$ is zero-mean, white, with covariance matrix :

$$
Q \stackrel{\operatorname{def}}{=} E\left[\varepsilon_{k} \varepsilon_{k}^{T}\right]
$$

where $E($.$) denotes the expectation operator. The mod-$ al characteristics defined in Equation (2) are equivalently found in the eigenstructure $\left(\lambda, \varphi_{\lambda}\right)$ of $F$ :

$$
e^{\tau \mu}=\lambda, \psi_{\mu}=\phi_{\lambda} \stackrel{\text { def }}{=} H \varphi_{\lambda} .
$$

From now on, we consider linear multi-variable systems described by the state space model given by Equation (3), where state $X$ and observed output $Y$ have dimensions $2 m$ and $r$ respectively. The state noise process $\left(\varepsilon_{k}\right)_{k}$ is an unmeasured Gaussian white noise sequence with zero mean and constant covariance matrix $Q$.

\section{Subspace identification methods}

We consider linear multi-variable systems described by a discrete-time state space model:

$$
\left\{\begin{array}{l}
X_{k+1}=F X_{k}+\varepsilon_{k} \\
Y_{k}=H X_{k}+v_{k}
\end{array}\right.
$$

where state $X$ and observed output $Y$ have dimensions $2 m$ and $r$ respectively. The number of degrees of freedom is $m$ and $r$ is the number of sensors.

The state noise process $\left(\varepsilon_{k}\right)_{k}$ is an unmeasured Gaussian white noise sequence with zero mean. We 
assume noise $\varepsilon_{k}$ to be stationary that is of constant covariance matrix; the issue of robustness with respect to non-stationary excitation is addressed in detail in the papers by Mevel et al. (2003) and Basseville et al. (2000).

The measurement noise process $\left(v_{k}\right)_{k}$ is assumed to be an unmeasured Moving Average process denoted by MA(i), a Gaussian sequence with zero mean. In the sequel, we use the notational convention that $i=-1$ for no measurement noise, and $i=0$ for white measurement noise. Note that, with this MA assumption for its structure, measurement noise does not affect the eigenstructure of the system given by Equation (8).

Let:

$$
G \stackrel{\operatorname{def}}{=} E\left[X_{k} Y_{k}^{T}\right]
$$

be the cross-correlation between state $X_{k}$ and observation $Y_{k}$, and let:

$$
O_{p}=\left[\begin{array}{c}
H \\
H F \\
H F^{2} \\
\vdots \\
H F^{p-1}
\end{array}\right] \text { and } C_{p}=\left[\begin{array}{lllll}
G & F G & F^{2} G & \cdots & F^{p-1} G
\end{array}\right] \text {, }
$$

be the $p^{\text {th }}$-order observability matrix of the system given by Equation (8) and controllability matrix of pair (F, $\mathrm{G})$, respectively. We assume that, for $p$ large enough, both observability and controllability matrices have full rank $2 m$.

Consider now a sequence of covariances:

$$
R_{j} \stackrel{\text { def }}{=} E\left(Y_{k+j} Y_{k}^{T}\right)
$$

of output $Y_{k}$ of a state space model represented by Equation (8). For $q>p+1$, let $H_{p+1, \mathrm{q}}$ be the blockHankel matrix:

$$
H_{p+1, q}=\left[\begin{array}{cccc}
R_{i+1} & R_{i+2} & \cdots & R_{i+q} \\
R_{i+2} & R_{i+3} & \cdots & R_{i+q+1} \\
\vdots & \vdots & \ddots & \vdots \\
R_{i+p+1} & \cdots & \cdots & R_{i+p+q}
\end{array}\right] .
$$

As mentioned above, integer $i$ reflects the assumed dependency in the measurement noise sequence $\left(v_{k}\right)_{k}$. It should be considered as a design parameter for the algorithms described in the paper.

Choosing the eigenvectors of $F$ as a basis for the state space of model of Equation (8) yields the following particular representation of the observability matrix introduced in Equation (10a) (Basseville et al. 2000):

$$
O_{p+1}(\theta)=\left(\begin{array}{c}
\phi \\
\phi \Delta \\
\vdots \\
\phi \Delta^{p}
\end{array}\right)
$$

where diagonal matrix $\Delta$ is defined as $\Delta=\operatorname{diag}(\lambda)$, and $\Lambda$ and $\phi$ are as defined in Equation $(7 \mathrm{a}, \mathrm{b})$. For any other state basis, the observability matrix $O_{p+1}$ can be written as:

$$
O_{p+1}=O_{p+1}(\theta) U
$$

for a suitable $2 m \times 2 m$ invertible matrix $U$. Because of the definition of $H_{p+1, q}, O_{p}$ and $C_{q}$ in Equation (12) and Equation (10 a, b) respectively, a direct computation of the $R_{j}$ 's from the model equations :

$$
R_{i+j+1}=H F^{i+j+1} G \quad(j \geq 0)
$$

leads to the following well known factorization property:

$$
H_{P+1, q}=O_{p+1}\left(F^{i+1} C_{q}\right) .
$$

Let $W 1, W 2$ be two user-defined invertible weighting matrices of size $(p+1) r$ and $q r$, respectively. Let:

$$
W_{1} H_{p+1, q} W_{2}^{T}=\left(\begin{array}{ll}
p & p^{\perp}
\end{array}\right)\left(\begin{array}{cc}
D & 0 \\
0 & 0
\end{array}\right) V^{T}
$$

be the Singular Value Decomposition (SVD) of this matrix, where $\mathrm{D}=\operatorname{diag}(\sigma 1, \ldots, \sigma 2 m)$ contains the $2 m$ non-zero singular values in decreasing order, the $2 \mathrm{~m}$ columns of matrix $\mathrm{P}$ are the corresponding left singular vectors, and the $(q r-2 \mathrm{~m})$ columns of matrix $p^{\perp}$ are the left singular vectors associated with singular value 0 . Note that $P$ is full column rank (f.c.r.). The factorization property of the Hankel matrix Equation (16) results in:

$$
W_{1} H_{p+1, q} W_{2}^{T}=W_{1} O_{p+1}\left(\begin{array}{ll}
F^{i+1} & C_{q}
\end{array}\right) W_{2}^{T}
$$

with, for example: $W_{1} O_{p+1}=P D^{1 / 2}$, where $T$ is a nonsingular transformation. The observability matrix can then be recovered, up to a change of basis or equivalently for some pair $(\mathrm{H}, \mathrm{F})$ as:

$$
O_{p+1}(H, F)=W_{1}^{-1} P D^{1 / 2} T .
$$

The particular choice of factor $D^{1 / 2}$, together with $T$ identity, corresponds to the balanced realization (BR) case when weighting matrices $W_{1}$ and $W_{2}$ are identity. Any other non-singular factor may be used.

The pair $(H, F)$ is recovered from $O_{p+1}(H, F)$ in a standard way (Peeters, Ventura 2003). The eigenstruc- 
ture of the state transition matrix $F$ is then recovered from: $F=\phi \Delta \phi^{-1}$, obtained, e.g., by eigenvalue solving.

In practice, one has only a limited data sample of a certain length and therefore only empirical correlations may be computed. The block Hankel matrix will consequently be generically of full rank. In this case, truncation of the SVD will be necessary. The estimated observability matrix will no longer enjoy the shift structure and the state matrices will be only estimates of the true ones. This is one source of error that can be found in the identification results. Therefore the Balanced Realization Algorithm (BR) is employed.

\section{Numerical examples}

\subsection{The tripod simplex}

The tripod simplex tensegrity system shown in Figure 1 is chosen as a case study. It is made up of six nodes whose coordinates are listed in Table 1, and twelve elements: nine cables and three struts. The boundary conditions were chosen in such a way to block the three lower nodes 1, 2 and 3 in all directions ( $X, Y$ and $Z$ ). Hence, the structure has nine degrees of freedom de-

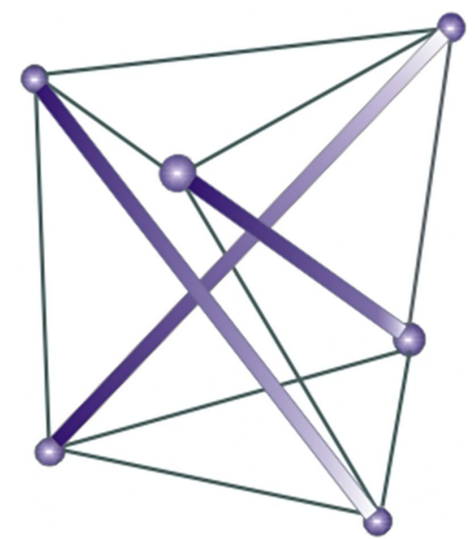

Fig. 1. The tripod simplex structure fined as follows: three degrees of freedom per upper node, respectively for nodes 4,5 and 6 and in the order $X, Y$ then $Z$. The three lower cables become redundant and can be omitted, since they do not contribute to the system dynamic characteristics. The cable elements have an elastic modulus of elasticity of $0.410^{11} \mathrm{~N} / \mathrm{m}^{2}$, a cross-section area of $0.2810^{-4} \mathrm{~m}^{2}$, a unit weight of $2.05 \mathrm{~N} / \mathrm{m}$ and an unstretched length of $1 \mathrm{~m}$. The struts have an elastic modulus of elasticity of $0.210^{12} \mathrm{~N} / \mathrm{m}^{2}$, a cross-section area of $3.2510^{-4} \mathrm{~m}^{2}$ and a unit weight of $27.9 \mathrm{~N} / \mathrm{m}$.

Table 1.Initial coordinates of the tripod simplex structure

\begin{tabular}{|c|c|c|c|}
\hline Node & $X(m)$ & $Y(m)$ & $Z(m)$ \\
\hline 1 & 0.5774 & 0.00 & 0.00 \\
\hline 2 & -0.2444 & 0.5000 & 0.00 \\
\hline 3 & -0.2665 & -0.4617 & 0.00 \\
\hline 4 & -0.4523 & 0.3015 & 0.9194 \\
\hline 5 & 0.94 & -0.5424 & 0.9194 \\
\hline 6 & 0.5094 & 0.2793 & 0.9194 \\
\hline
\end{tabular}

The system in its initial configuration has one infinitesimal mechanism and a single state of self-stress. An initial pretension of its components is needed to stabilize it. Thus, a geometric nonlinear analysis was performed to determine the pre-stressed geometry of the simplex tripod. Once equilibrium is reached, the nine natural frequencies of vibration of this structure and their corresponding mode shapes were determined. The resulting natural frequencies of the system are listed in Table 2 and referred to as exact.

Mode 1, shown in Fig. 3a, corresponds to a rotation of the upper triangular cables in the horizontal plane with respect to the centroïdal axis of the tripod simplex. It is a torsion accompanied with a stretch- a)

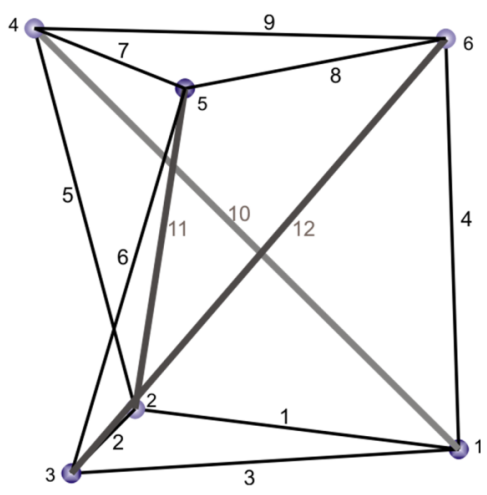

b)

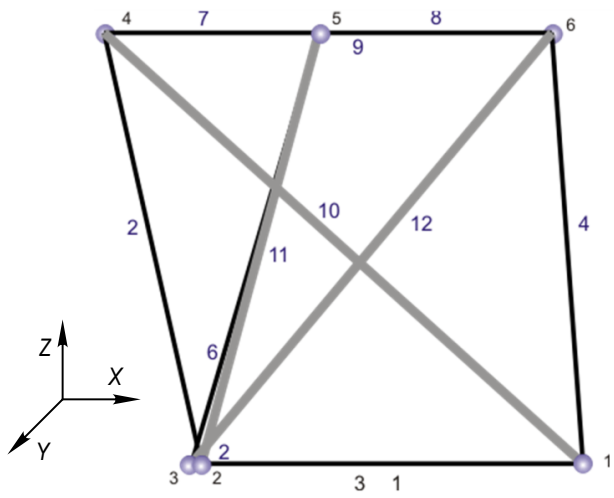

c)

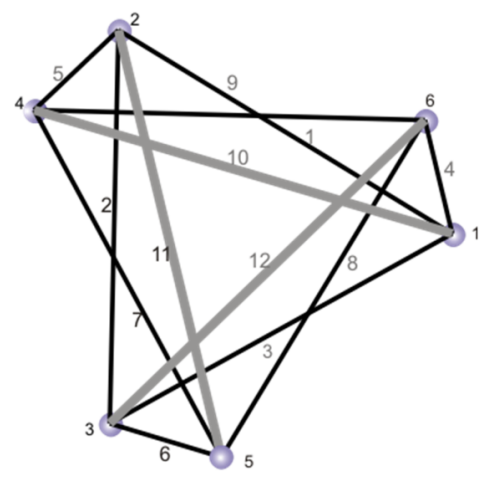

Fig. 2. The stabilized tripod simplex (a) General view( b) front view(c) Top view 
Table 2. The exact and identified natural frequencies of the tripod simplex structure

\begin{tabular}{|c|c|c|c|c|c|c|c|c|}
\hline \multirow{3}{*}{ Mode } & \multirow{3}{*}{$\begin{array}{c}\omega \text { exact } \\
(\mathrm{rad} / \mathrm{s})\end{array}$} & \multicolumn{7}{|c|}{$\omega \mathrm{id} .(\mathrm{rad} / \mathrm{s})$} \\
\hline & & \multicolumn{7}{|c|}{ Sensors (locations) } \\
\hline & & 9 & $\begin{array}{c}8 \\
(12345678)\end{array}$ & $\left.\begin{array}{c}7 \\
(1234567\end{array}\right)$ & $\begin{array}{c}6 \\
(123456)\end{array}$ & 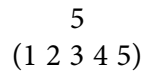 & $\begin{array}{c}4 \\
(1358)\end{array}$ & $\begin{array}{c}3 \\
(347)\end{array}$ \\
\hline 1 & 6.723 & 6.688 & 6.575 & 6.7103 & 6.733 & 6.852 & 6.711 & 6.553 \\
\hline 2 & 57.194 & 57.060 & 56.999 & 56.164 & 55.791 & 55.932 & 56.564 & 57.546 \\
\hline 3 & 57.197 & 57.673 & 57.192 & 56.917 & 57.112 & 57.123 & 57.577 & \\
\hline 4 & 131.217 & 130.685 & 129.835 & 129.685 & 129.491 & 129.002 & 127.848 & 127.371 \\
\hline 5 & 131.219 & 131.261 & 131.908 & 131.234 & 133.289 & 130.124 & 132.725 & 129.469 \\
\hline 6 & 156.611 & 156.800 & 156.242 & 156.294 & 157.106 & 156.027 & 157.506 & 151.489 \\
\hline 7 & 755.773 & 756.168 & 750.265 & 744.163 & & & 755.309 & 750.766 \\
\hline 8 & 755.790 & 756.260 & 755.077 & 755.262 & 756.324 & 755.016 & 762.064 & 755.910 \\
\hline 9 & 766.354 & 766.832 & 767.444 & 763.797 & 763.518 & 764.262 & 766.525 & 765.711 \\
\hline
\end{tabular}

ing of the element. For mode 2 (see Fig. 3b), a rotation of the upper triangular cables with respect to an axis parallel to the direction of the seventh cable and intersecting mid-spans of the eighth and ninth cables is observed. Similar deformations are obtained for the third mode (Fig. 3c) as for the second one which occurs at almost the same natural frequency, thus a rotation of the upper triangular cables with respect to an axis parallel to the direction of the eighth cable and intersecting mid-spans of the seventh and ninth cables is observed. The fourth mode (Fig. 3d) is characterized by a deformation of the upper triangular cables in such a way that when the eighth cable is stretched, cables number 7 and 9 are shortened and vice versa when the seventh and ninth cables are elongated, cable number 8 is shortened. These deformations are accompanied with alternate rotations of the upper triangular cables with respect to the rotational axes characterizing the second and third modes. Similar deformations are obtained for the fifth mode (Fig. 3e) as for the fourth one which occurs at almost the same natural frequency, thus the fifth mode characterized by a deformation of the upper triangular cables in such a way that when the ninth cable is stretched, cables number 7 and 8 are shortened and vice versa when the seventh and eighth cables are elongated, cable number 9 is shortened, and the same rotations are observed. In the sixth mode (Fig. 3f), the upper triangular cables shorten (shrinkage) as the simplex stretches vertically along the $\mathrm{Z}$ axis and elongate (expansion) as the simplex shortens vertically. For mode 7 (Fig. 3g), a rotation of the upper triangular cables with respect to an axis parallel to the direction of the seventh cable and intersecting mid-spans of the eighth and ninth cables is observed. This rotation is followed by a deformation of the upper triangular cables in such a way that when the seventh cable is stretched, cables number 8 and 9 are shortened and vice versa when the eighth and ninth cables are elongated, cable number 7 is shortened. Similar deformations are obtained for the eighth mode (Fig. 3g) as for the seventh one which occurs a)

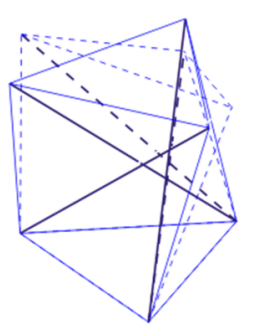

d)

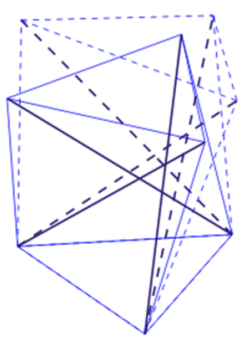

g)

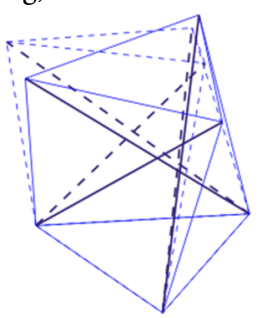

b)

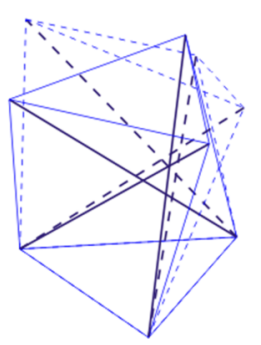

e)

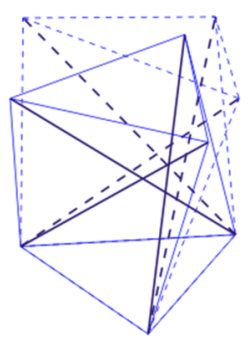

h)

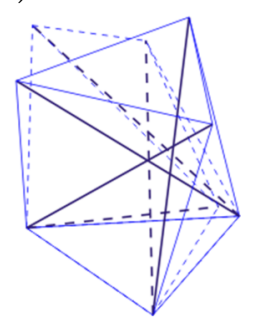

c)

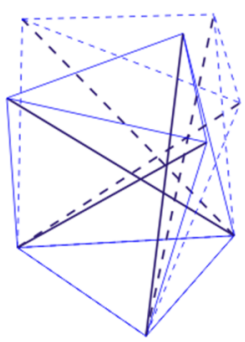

f)

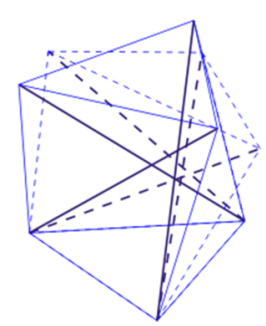

i)

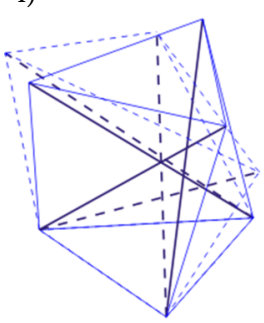

Fig.3. The nine mode shapes of the Tripod simplex structure 
at almost the same natural frequency, thus a rotation of the upper triangular cables with respect to an axis parallel to the direction of the ninth cable and intersecting mid-spans of the seventh and eighth cables is observed. This rotation is followed by a deformation of the upper triangular cables in such a way that when the ninth cable is stretched, cables number 7 and 8 are shortened and vice versa when the seventh and eighth cables are elongated, cable number 9 is shortened. In the ninth mode (Fig. 3h), the upper triangular cables elongate (expansion) as the simplex stretches vertically along the $\mathrm{Z}$ axis and shorten (shrinkage) as the simplex shortens vertically.

The structure is excited using random forces. A total of 30000 data points were generated at a sampling frequency of $4000 \mathrm{~Hz}$. 2\% noise is added to the outputs in order to simulate measurement noise.

There exist several implementations of the stochastic subspace method; one of these is the Balanced Realization Algorithm (BR). This method is based on the SVD decomposition of the Hankel matrix $H_{p+1, q}$ associated with the state system of Equation (8).

Practical experience with real data as investigated by (Reynders et al. 2007) showed that it is better to over specify the model order and to eliminate spurious numerical poles afterwards. This can be done by constructing stabilization diagrams. By rejecting less singular values, models of increasing order are determined. Each model yields a set of modal parameters and these can be plotted in a stabilization diagram. The criteria used are $1 \%$ for eigenfrequencies, $5 \%$ for damping ratios and $99 \%$ for mode shape vectors (MAC). A mode (frequency, damping and modal shape) is considered stable if it satisfies these three criteria.
The stabilization diagram of a multi-patch trial with all the sensors is implemented. Since all the available sensors are used, the animated mode shapes can be plotted.

Different sensor locations have been studied to identify the nine mode shapes of the tripod simplex and their corresponding natural frequencies and damping ratios. Table 2 shows the values of the exact and identified natural frequencies for each sensor pool. Table 3 shows the values of the exact and identified damping ratio for different sensor pools.

The inspection of these two tables indicates that the identified natural frequencies and damping ratios for most sensor pools were well identified. However, in the case when six sensors placed along degrees of freedom 1, 2, 3, 4, 5 and 6 and the case when five sensors placed along degrees of freedom 1, 2, 3, 4 and 5 were used, mode seven was not identified. It is to be noted that mode seven occurs at almost the same frequency as mode 8 and displays symmetric deformation shape. This was also observed for the case when three sensors placed along degrees of freedom 3, 4 and 7 were used, mode three was not identified. This mode occurs at almost the same frequency as mode 2 and displays symmetric deformation shape. This may be attributed to identifiably conditions which is known to be dependent on the sensor locations (this issue is not treated in this paper) and to the persistency of the excitation. Since it is difficult to separate these close modes based only on frequencies, we have introduced in this work the use of the MAC value in the stabilization diagram in order to separate the modes. The MAC between the exact and identified mode shapes of the tripod simplex structure are presented in Table 4 . The inspection of

Table 3. The exact and identified damping ratios of the tripod simplex structure

\begin{tabular}{|c|c|c|c|c|c|c|c|c|}
\hline \multirow{3}{*}{ Mode } & \multirow{3}{*}{$\begin{array}{c}\varsigma \text { exact } \\
(\%)\end{array}$} & \multicolumn{7}{|c|}{ sid. (\%) } \\
\hline & & \multicolumn{7}{|c|}{ Sensors (locations) } \\
\hline & & 9 & $\begin{array}{c}8 \\
(12345678)\end{array}$ & $\begin{array}{c}7 \\
(1234567)\end{array}$ & $\begin{array}{c}6 \\
(123456)\end{array}$ & 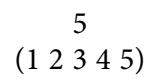 & $\begin{array}{c}4 \\
(1358)\end{array}$ & $\begin{array}{c}3 \\
(347)\end{array}$ \\
\hline 1 & 5.00 & 5,12 & 5,78 & 4,93 & 4,39 & 4,86 & 3,06 & 4,79 \\
\hline 2 & 5.00 & 4,87 & 5,12 & 4,43 & 3,50 & 5,45 & 6,87 & 4,77 \\
\hline 3 & 5.00 & 5,12 & 4,26 & 4,85 & 5,85 & 3,15 & 4,39 & \\
\hline 4 & 5.00 & 5,16 & 5,51 & 5,89 & 7,44 & 4,91 & 7,47 & 4,59 \\
\hline 5 & 5.00 & 7,58 & 4,76 & 4,95 & 3,82 & 7,70 & 5,69 & 6,32 \\
\hline 6 & 5.00 & 5,64 & 4,84 & 5,64 & 6,96 & 5,77 & 5,75 & 4,85 \\
\hline 7 & 5.00 & 4,86 & 5,23 & 6,20 & & & 4,86 & 5,74 \\
\hline 8 & 5.00 & 4,67 & 4,70 & 5,01 & 5,29 & 5,10 & 5,37 & 5,07 \\
\hline 9 & 5.00 & 5,83 & 5,26 & 5,11 & 5,01 & 5,48 & 5,28 & 5,41 \\
\hline
\end{tabular}


Table 4. MAC between the exact and identified mode shapes of the tripod simplex structure

\begin{tabular}{|c|c|c|c|c|c|c|c|c|}
\hline \multirow{4}{*}{ Mode } & \multirow{4}{*}{$\begin{array}{c}\omega \text { exact } \\
(\mathrm{rad} / \mathrm{s})\end{array}$} & \multicolumn{7}{|c|}{ Mac (identified mode - exact mode) } \\
\hline & & \multicolumn{7}{|c|}{ Sensors (locations) } \\
\hline & & \multirow[t]{2}{*}{9} & 8 & 7 & 6 & 5 & 4 & 3 \\
\hline & & & $(12345678)$ & $(1234567)$ & $(123456)$ & $\left(\begin{array}{lllll}1 & 2 & 3 & 4 & 5\end{array}\right)$ & $\left(\begin{array}{llll}1 & 3 & 5 & 8\end{array}\right)$ & $\left(\begin{array}{lll}3 & 4 & 7\end{array}\right)$ \\
\hline 1 & 6.723 & 99.99 & 99.99 & 99.99 & 99.99 & 99.99 & 99.99 & 99.99 \\
\hline 2 & 57.194 & 97.86 & 89.80 & 85.01 & 97.09 & 94.17 & 95.73 & 99.58 \\
\hline 3 & 57.197 & 99.69 & 95.10 & 92.55 & 98.24 & 98.81 & 85.90 & \\
\hline 4 & 131.217 & 97.23 & 91.64 & 97.26 & 98.76 & 95.42 & 98.20 & 98.77 \\
\hline 5 & 131.219 & 97.09 & 97.60 & 92.55 & 92.46 & 81.77 & 85.94 & 85.90 \\
\hline 6 & 156.611 & 99.87 & 99.84 & 99.95 & 99.28 & 99.87 & 98.99 & 84.73 \\
\hline 7 & 755.773 & 90.04 & 98.34 & 97.04 & & & 94.26 & 94.52 \\
\hline 8 & 755.790 & 93.91 & 96.73 & 98.60 & 95.01 & 98.54 & 86.49 & 85.67 \\
\hline 9 & 766.354 & 99.22 & 96.79 & 96.17 & 99.30 & 98.23 & 99.46 & 88.89 \\
\hline
\end{tabular}

this table reveals that in general, the mode shapes were well identified except for the close modes and depending on sensor locations.

For the damping ratios however, the identified values were less accurate than for the frequencies. This observation is inherent to the stochastic identification algorithms which were reported in the literature (Magalhães et al. 2009).

In order to validate the identified modal parameters, model based data correlations were computed and compared to the empirical ones (or those equivalently the spectra). The sensors 3,4 and 7 were used to identify the experimental natural frequencies, damping ratios, mode shapes and correlations from the first 30000 data samples. The superposed correlations are shown in Figure 4. The inspection of this figure indicates that the modal model reproduces well the empirical correlations and this may be attributed to the fact that most of the modes were well identified.

A second procedure to validate the system identification is the spectral analysis. Three spectra are presented in Fig. 5. They are compared with those obtained by applying FFT to measurements. From these comparisons, it's shown that the structure model parameters are well identified since model and data spectrum fit in spite of the divergence in the auto spectrum of the sensor 3. In fact, the peaks in this Figures ( $5 \mathrm{a}$, b, c) are better identified than the valleys. This can be understood from the implementation of the CovSSI method that doesn't guarantee the positive real sequence condition (Van Overchee, De Moor 1996). Therefore, the extended covariance matrices might not be positive. Thus, the spectrum divergences are well justified. a)
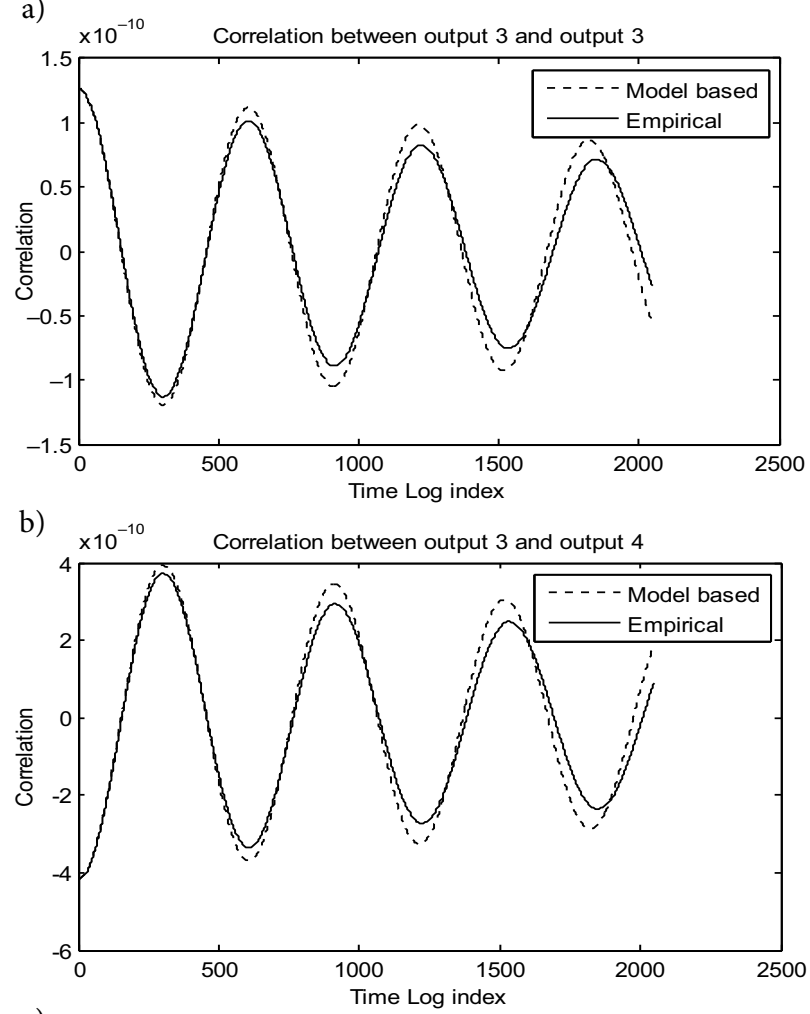

c)

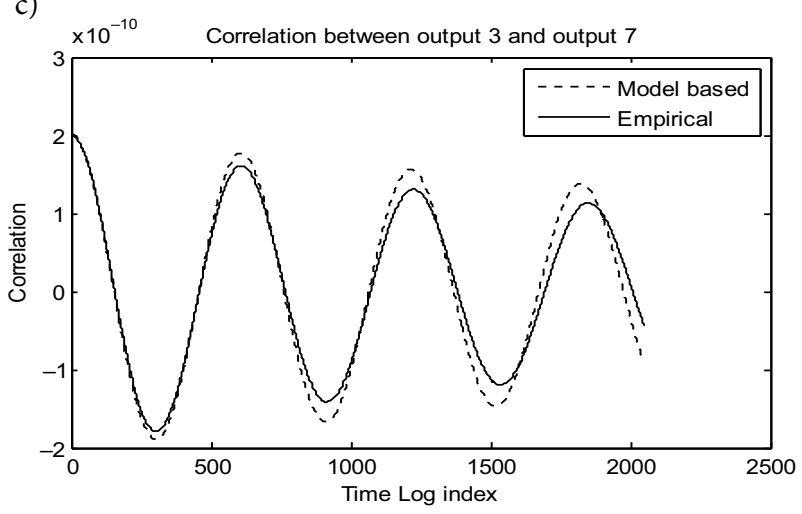

Fig. 4. Empirical correlations vs. model based ones of the Tripod simplex structure 
a)

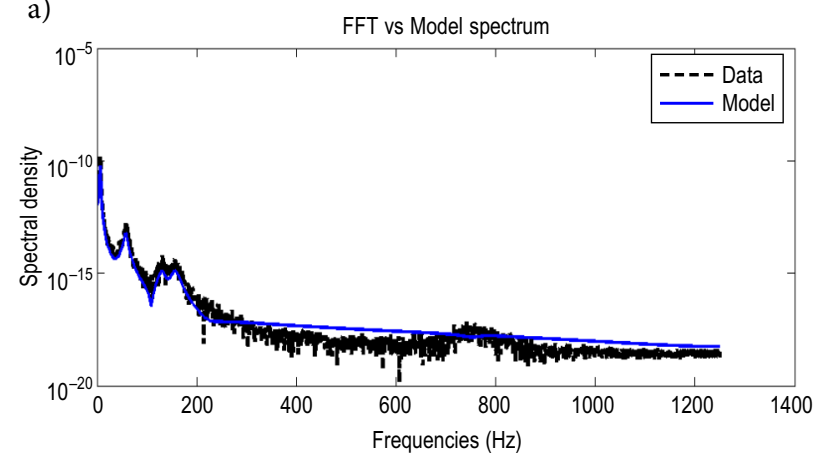

b)

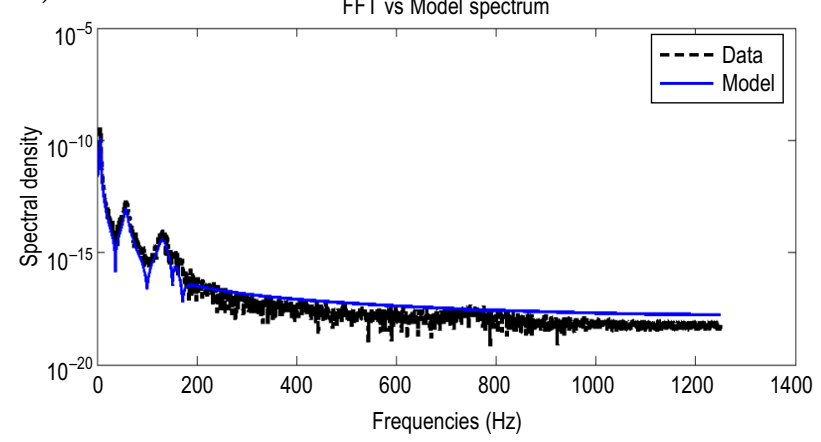

c)

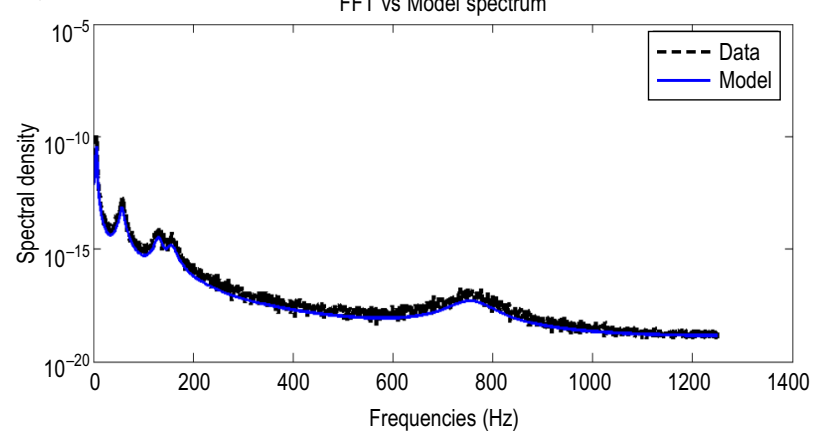

Fig. 5. Cross spectrum of the Tripod simplex structure (a) Auto spectrum sensor 3, (b) Cross spectrum between 3 and 4, (c) Cross spectrum between 3 and 7

\subsection{Geiger dome}

The second example considered is a small scale tensegrity dome of a Geiger type. The model of the Geiger type structure (El Ouni, Ben Kahla 2014) is shown in Figure 6.

The small scale tensegrity dome of a Geiger type is made up of 32 nodes and 73 elements: 13 vertical struts (numbered from 1 to 13 ) and 60 strings (numbered from 14 to 73 ). The boundary conditions were chosen in such a way to restrain the motion of nodes $7,12,17,22,27$ and 32 in all directions $(X, Y$ and $Z)$. Hence, the structure has 78 degrees of freedom defined as follows: three degrees of freedom per inner node in

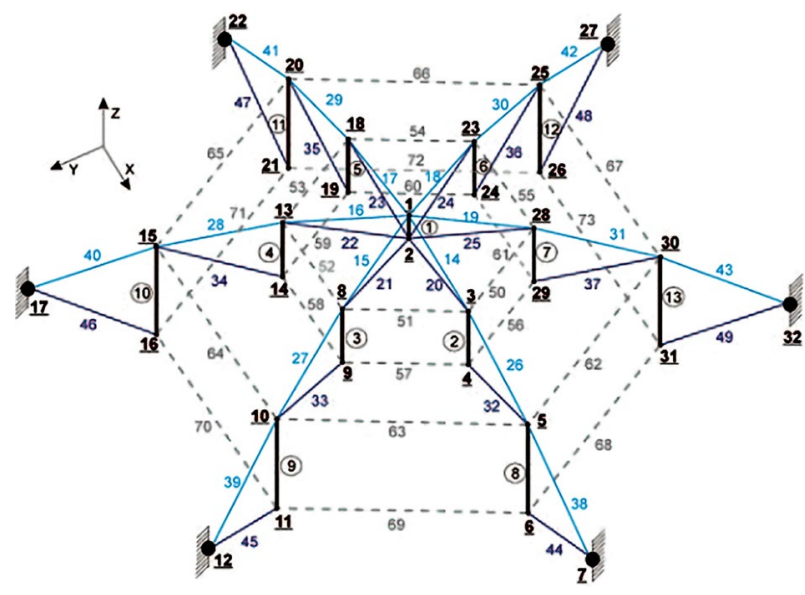

Fig. 6. The tensegrity dome of a Geiger type

the order $X, Y$ then $Z$. The coordinates of all nodes are given in Table 5.

All the elements have an elastic modulus of elasticity of $1.610^{11} \mathrm{~N} / \mathrm{m}^{2}$ and a density of $7.8510^{3} \mathrm{Kg} / \mathrm{m}^{3}$. The cross-section area of the struts is equal to $10^{-4} \mathrm{~m}^{2}$. The cross-section area and initial pre-tension of cable elements are given in Table 6 . The structure is excited by a white noise. Only five sensors measurements and a sampling frequency $f_{s}=30 \mathrm{~Hz}$ are available for the identification.

The same spectral analysis procedure used for the tripod simplex structure to validate system identification is employed for the Geiger dome. In Figures 7-11, five spectra are presented and compared with the spectra obtained by the application of FFT to measurements. These comparisons show clearly that the parameters of the structure model are well identified since the data spectra and the model fit despite the divergence that appeared in all the cross spectrum and the autospectrum of sensor 3.

By inspecting Figure 9, we clearly notice that all valleys and peaks are well superposed except the valley that is close to the Nyquist frequency $(15 \mathrm{~Hz})$. These facts enable us to conclude that peaks are better identified than the valleys in all data and model spectra. In fact, we can understand that from the implementation of the SSI-cov method through which a positive real sequence condition (Basseville et al. 2000) cannot be guaranteed. For that reason, we might not have positive extended covariance matrices. As a result, the spectrum divergences in that case, are well justified. 
Table 5. Initial coordinates of the smart Geiger's dome

\begin{tabular}{|c|c|c|c|c|c|c|c|}
\hline Node $^{\circ}$ & $X(\mathrm{~m})$ & $Y(\mathrm{~m})$ & $Z(\mathrm{~m})$ & Node $\mathrm{N}^{\circ}$ & $X(\mathrm{~m})$ & $Y(\mathrm{~m})$ & $Z(\mathrm{~m})$ \\
\hline 1 & 0 & 0 & 0.21 & 17 & -0.30 & 0.519615 & 0 \\
\hline 2 & 0 & 0 & 0.15 & 18 & -0.20 & 0 & 0.185 \\
\hline 3 & 0.20 & 0 & 0.185 & 19 & -0.20 & 0 & 0.45 \\
\hline 4 & 0.20 & 0 & 0.45 & 20 & -0.40 & 0 & 0.115 \\
\hline 5 & 0.40 & 0 & 0.115 & 21 & -0.40 & 0 & -0.115 \\
\hline 6 & 0.40 & 0 & -0.115 & 22 & -0.60 & 0 & 0 \\
\hline 7 & 0.60 & 0 & 0 & 23 & -0.10 & -0.173205 & 0.185 \\
\hline 8 & 0.10 & 0.173205 & 0.185 & 24 & -0.10 & -0.173205 & 0.45 \\
\hline 9 & 0.10 & 0.173205 & 0.45 & 25 & -0.20 & -0.346410 & 0.115 \\
\hline 10 & 0.20 & 0.346410 & 0.115 & 26 & -0.20 & -0.346410 & -0.115 \\
\hline 11 & 0.20 & 0.346410 & -0.15 & 27 & -0.30 & -0.519615 & 0 \\
\hline 12 & 0.30 & 0.519615 & 0 & 28 & 0.10 & -0.173205 & 0.185 \\
\hline 13 & -0.10 & 0.173205 & 0.185 & 29 & 0.10 & -0.173205 & 0.45 \\
\hline 14 & -0.10 & 0.173205 & 0.45 & 30 & 0.20 & -0.346410 & 0.115 \\
\hline 15 & -0.20 & 0.346410 & 0.115 & 31 & 0.20 & -0.346410 & -0.115 \\
\hline 16 & -0.20 & 0.346410 & -0.115 & 32 & 0.30 & -0.519615 & 0 \\
\hline
\end{tabular}

Table 6. Cross-section area and initial pre-tension of different cable families of the Geiger dome

\begin{tabular}{|c|c|c|c|c|}
\hline Cable family & Cable number & Cross-section area $\left(10^{-4} \mathrm{~m}^{2}\right)$ & Initial pre-tension $(\mathrm{KN})$ & Initial pre-stress $(\mathrm{MPa})$ \\
\hline 1 & $14-19$ & 0.01 & 0.20 & 200 \\
\hline 2 & $20-25$ & 0.01 & 0.144 & 144 \\
\hline 3 & $26-31$ & 0.02 & 0.519 & 259.5 \\
\hline 4 & $32-37$ & 0.02 & 0.519 & 326 \\
\hline 5 & $38-43$ & 0.04 & 1.304 & 326 \\
\hline 6 & $44-49$ & 0.04 & 1.304 & 150 \\
\hline 7 & $50-55$ & 0.01 & 0.15 & 240 \\
\hline 8 & $56-61$ & 0.02 & 0.48 & 150 \\
\hline 9 & $62-67$ & 0.01 & 0.15 & 282.5 \\
\hline 10 & $68-73$ & 0.04 & 1.13 & \\
\hline
\end{tabular}

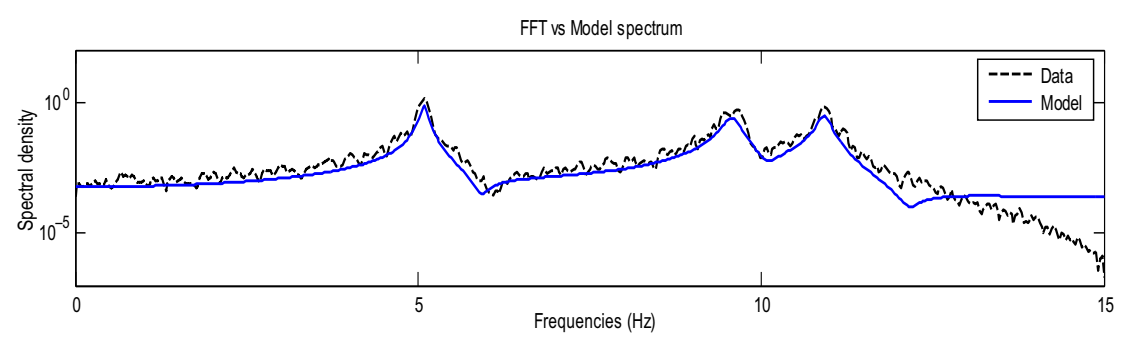

Corrélations Empirique vs Théorique

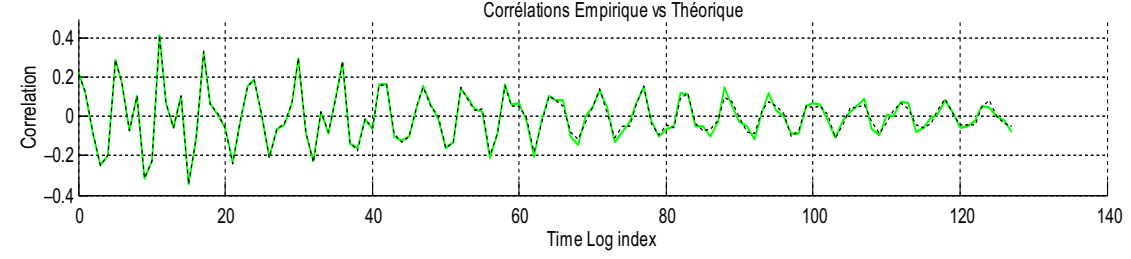

Fig. 7. Cross spectrum between sensors 1 and 5 of the Geiger dome 

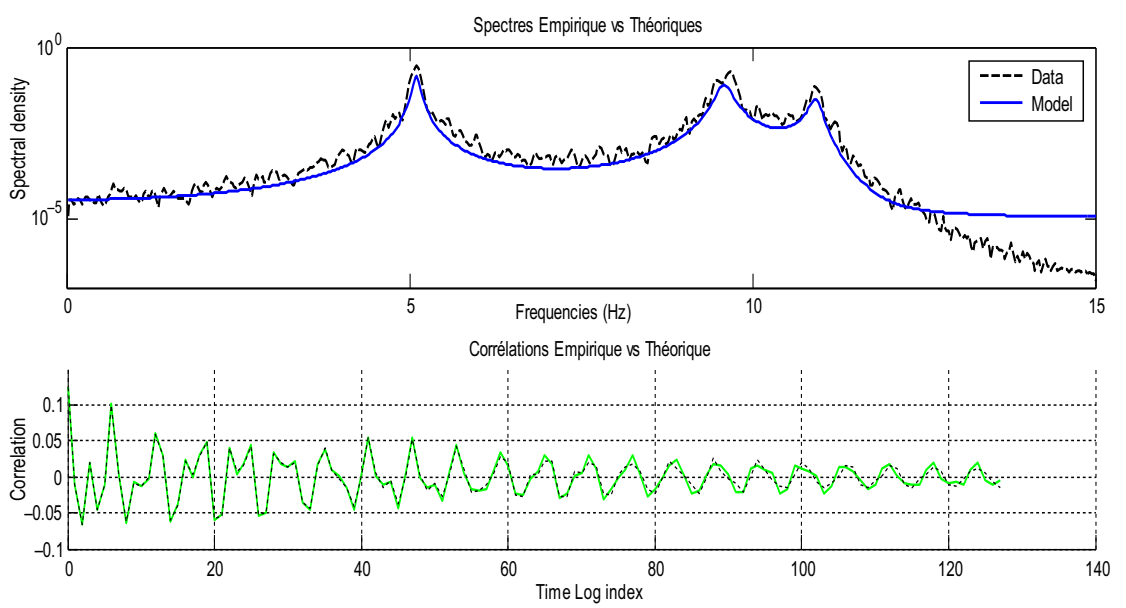

Fig. 8. Cross spectrum between sensors 5 and 20 of the Geiger dome
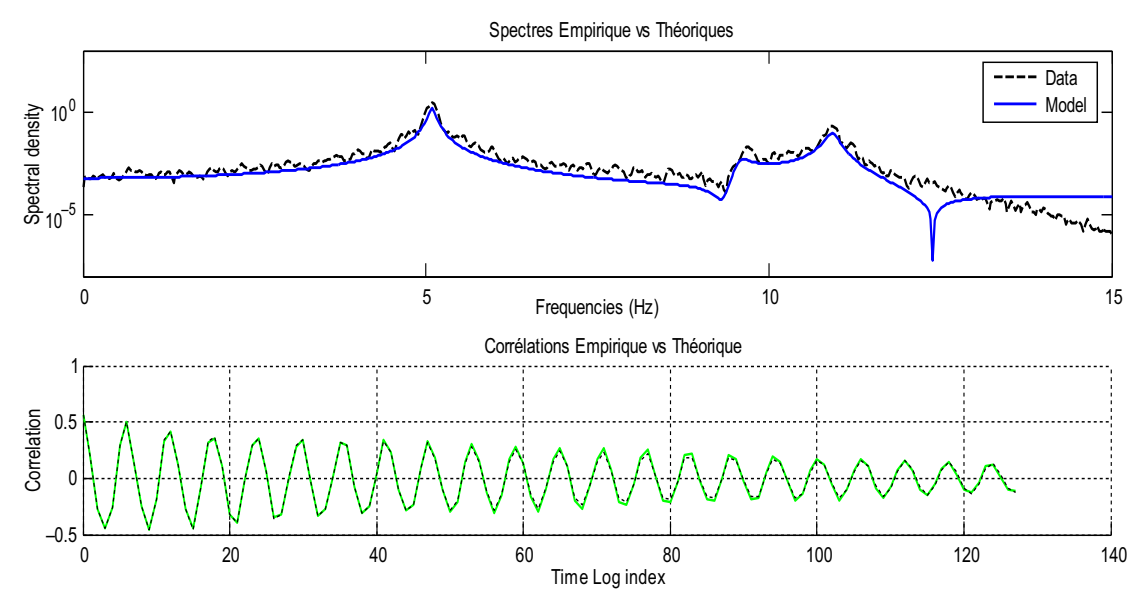

Fig. 9. Autospectrum sensor 3 of the Geiger dome
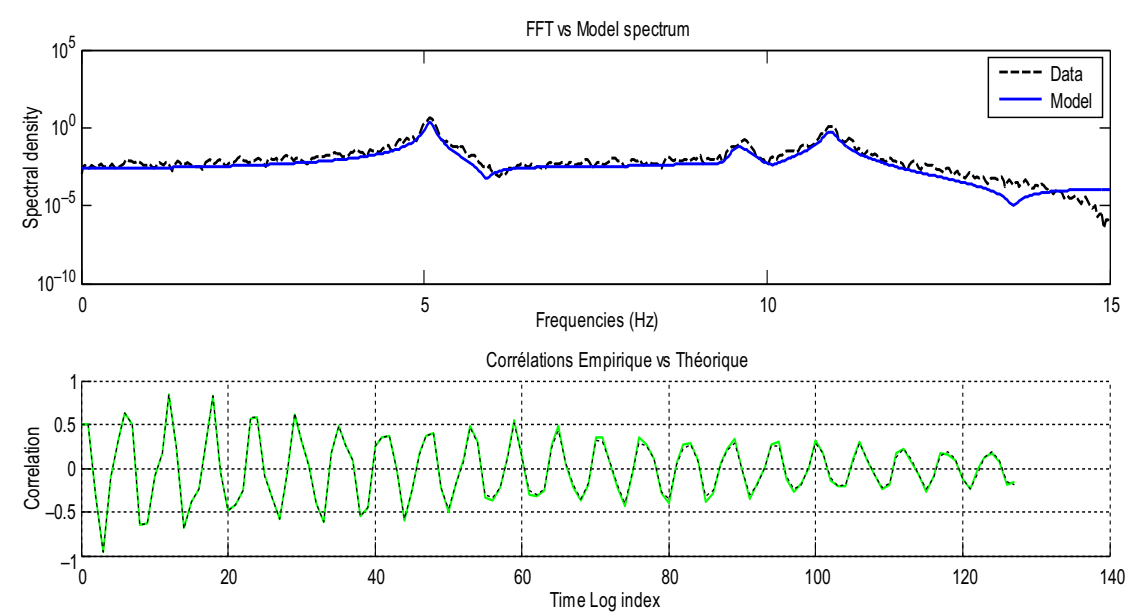

Fig. 10. Cross spectrum between sensors 1 and 3 of the Geiger dome

\section{Conclusions}

For tensegrity systems the structure modal signature is directly related to its state of self-stress. Thus identifying the modal parameters and monitoring their possible changes would result in the evaluation of the system internal forces and the possible detection of possible damages of its components. A simplex tensegrity structure with closely-spaced modes and a Geiger dome are considered here as an output-only 


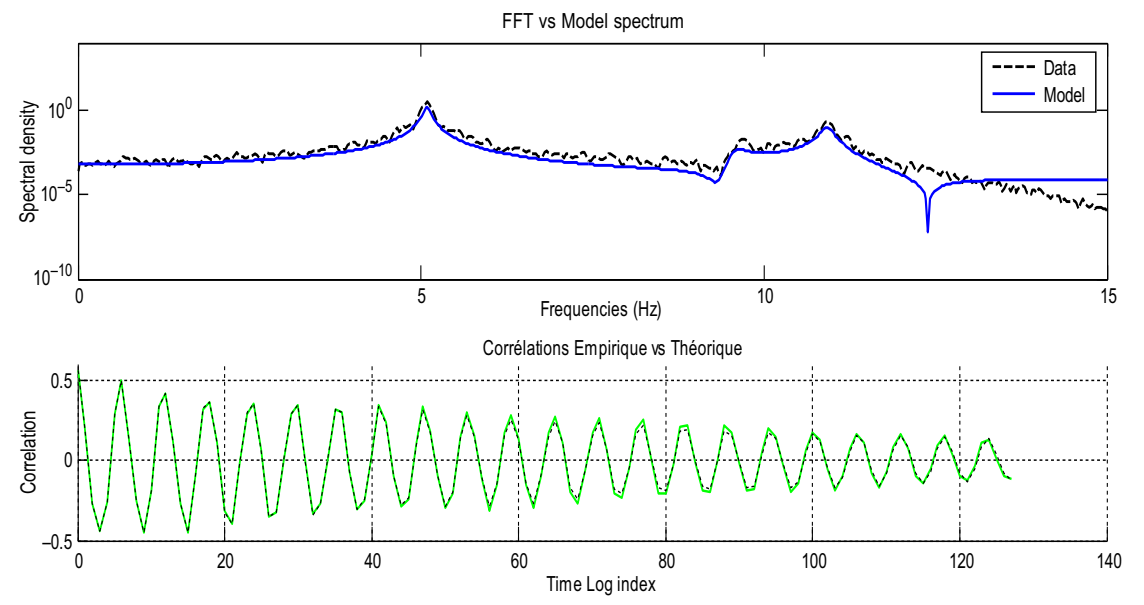

Fig. 11.Cross spectrum between sensors 3 and 18 of the Geiger dome

modal identification case studies. The Balanced Realization Algorithm has been investigated for the outputonly modal identification task. Different sensor locations have been studied to identify the mode shapes of tensegrity systems and their corresponding natural frequencies and damping ratios. Although the structures are considered stationeries, the BR algorithm performed quite well regarding the fact that the structure presents symmetries and therefore closely-spaced modes. The stabilization diagram was necessary to identify close modes by imposing a high value for the MAC. When a limited number of sensors are used, it is noticed that close modes were difficult to identify.

\section{References}

Arsenault, M.; Gosselin, C. M. 2006. Kinematic, static, and dynamic analysis of a spatial three-degree-of-freedom tensegrity mechanism, Journal of Mechanical Design 128(5): 10611069. http://dx.doi.org/10.1115/1.2218881

Basseville, M. et al. 2000. Subspace-based fault detection algorithms for vibration monitoring, Automatica 36(1): 101-109. http://dx.doi.org/10.1016/S0005-1098(99)00093-X

Basseville, M., et al. 2000. Output-only subspace-based structural identification: from theory to industrial testing practice, Journal of Dynamic Systems Measurement and Control transactions of The ASME 123(4).

Bel Hadj Ali, N.; Smith, I. F. C. 2009. Dynamic analysis and vibration control of an active tensegrity structure, in J. Rodellar, E. Reithmeier (Eds.). 15th International Workshop on Dynamics and Control. Barselona.

Bel Hadj Ali, N.; Smith, I. F. C. 2010. Dynamic behaviour and vibration control of a tensegrity structure, International Journal of Solids and Structures 47(9): 1285-1296. http:// dx.doi.org/10.1016/j.ijsolstr.2010.01.012

Benveniste, A.; Fuchs, J. J. 1985. Single sample modal identification of a nonstationary stochastic process, IEEE Transactions on Automatic Control 30(1): 66-74.

http://dx.doi.org/10.1109/TAC.1985.1103787
Ben Kahla, N., et al. 2000. Nonlinear dynamic analysis of tensegrity systems, Journal of The International Association for Shell and Spatial Structures 41(132): 49-58.

Carden, E. P.; Mita, A. 2009. Challenges in developing confidence intervals on modal parameters estimated for large civil infrastructure with stochastic subspace identification. Structural Control \& Health Monitoring. http://dx.doi.org/10.1002/stc.358

Dubé, J. F., et al. 2008. Comparison between experimental tests and numerical simulations carried out on a tensegrity minigrid, Engineering Structures 30(7): 1905-1912. http://dx.doi.org/10.1016/j.engstruct.2007.12.010

d'Estrée Sterk, T. 2003. Using actuated tensegrity structures to produce a responsive architecture, in The 2003 Annual Conference of the Association for Computer Aided Design in Architecture, October 2003, Indianapolis, IN, USA, 85-93.

El Ouni, M. H.; Ben Kahla, N. 2014. Active tendon control of a Geiger dome, Journal of Vibration and Control 20(2): 241255. http://dx.doi.org/10.1177/1077546312458944

Graells Rovira, A; Mirats Tur, J. 2009. Control and simulation of a tensegrity-based mobile robot, Robotics and Autonomous Systems 57(5): 526-535. http://dx.doi.org/10.1016/j.robot.2008.10.010

Giraldo, D. F., et al. 2009. Modal identification through ambient vibration: comparative study, Journal of Engineering Mechanics 135(8): 759-770.

http://dx.doi.org/10.1061/(ASCE)0733-9399(2009)135:8(759)

Ingber, D. E. 1998. The architecture of life, Scientific American 278(1): 48-57. http://dx.doi.org/10.1038/scientificamerican0198-48

Juan, S. H.; Mirats Tur, J. M. 2008. A method to generate stable, collision free configurations for tensegrity based robots, in Proceedings of IEEE/RSJ International Conference on Intelligent Robots and Systems 3769-3774.

Korkmaz, S.; Bel Hadj Ali, N.; Smith, I .F. C. 2011. Determining control strategies for damage tolerance of an active tensegrity structure, Engineering Structures 33(6): 1930-1939. http://dx.doi.org/10.1016/j.engstruct.2011.02.031

Korkmaz, S.; Bel Hadj Ali, N.; Smith, I. F. C. 2012. Configuration of control system for deployment and damage tolerance of a tensegrity bridge, Advanced Engineering Informatics 26(1): 145-155. http://dx.doi.org/10.1016/j.aei.2011.10.002 
Magalhaes, F., et al. 2008. Operational modal analysis and finite element model correlation of the Braga Stadium suspended roof, Engineering Structures 30(6): 1688-1698. http://dx.doi.org/10.1016/j.engstruct.2007.11.010

Magalhães, F., et al. 2009. Online automatic identification of the modal parameters of a long span arch bridge, Mechanical Systems and Signal Processing 23(2): 316-329. http://dx.doi. org/10.1016/j.ymssp.2008.05.003

Masic, M.; Skelton, R. E. 2006. Selection of prestress for optimal dynamic/control performance of tensegrity structures, International Journal of Solids and Structures 43(7-8): 2110-2125. http://dx.doi.org/10.1016/j.ijsolstr.2005.06.066

Mevel, L., et al.2003. Stochastic subspace-based structural identification and damage detection- Application to the steelquake benchmark, Mechanical Systems and Signal Processing 17(1): 91-101. http://dx.doi.org/10.1006/mssp.2002.1544

Motro, R., et al. 1986. Static and dynamic analysis of tensegrity systems, in ASCE International Symposium on Shells and Spatial Structures, Computational Aspects. New York: Springer, 270-279.

Motro, R. 2003. Tensegrity: Structural systems for the future. UK.

Murakami, H. 2001a. Static and dynamic analyses of tensegrity structures. Part II. Quasi-static analysis, International Journal of Solids and Structures 38(20): 3615-3629. http://dx.doi.org/10.1016/S0020-7683(00)00233-X

Murakami, H. 2001b. Static and dynamic analyses of tensegrity structures. Part 1. Nonlinear equations of motion, International Journal of Solids and Structures 38(20): 3599-3613. http://dx.doi.org/10.1016/s0020-7683(00)00232-8
Sultan, C., et al. 2002. Linear dynamics of tensegrity structures, Engineering Structures 24(6): 671-685. http://dx.doi.org/10.1016/S0141-0296(01)00130-4

Peeters, B.; Ventura, C. E. 2003. Comparative study of modal analysis techniques for bridge dynamic characteristics, Mechanical Systems and Signal Processing 17(5): 965-988. http://dx.doi.org/10.1006/mssp.2002.1568

Puig, L., et al. 2010. A review on large deployable structures for astrophysics missions, Acta Astronautica 67(1-2): 12-26. http://dx.doi.org/10.1016/j.actaastro.2010.02.021

Paul, C., et al. 2006. Design and control of tensegrity robots for locomotion, IEEE Transactions on Robotics 22(5): 944-957. http://dx.doi.org/10.1109/TRO.2006.878980

Reynders, E., et al. 2007. Uncertainty bounds on modal parameters obtained from stochastic subspace identification, Mechanical Systems and Signal Processing 22(4): 948-969. http://dx.doi.org/10.1016/j.ymssp.2007.10.009

Skelton, R. E., et al. 2001. An introduction to the mechanics of tensegrity structures, in Proceedings of the 40th IEEE Conference on Decision and Control 4255: 4254-4259. Orlando, Florida USA. http://dx.doi.org/10.1109/cdc.2001.980861

Skelton, R. E.; de Oliveira, M. C. 2009. Tensegrity systems. Springer.

Van Overchee, P.; De Moor, B. 1996. Subspace identification for linear systems: theory, implementation, applications. The Netherlands: Kluwer Academic Publishers. http://dx.doi.org/10.1007/978-1-4613-0465-4

Miniar ATTIG. Currently a PhD student at the Dept of Civil Engineering of the National School of Engineering of Tunis (ENIT), Tunisia, from which she obtained a Master of Science in structural engineering. She is a member of the Laboratory of Systems and Applied Mechanics, Polytechnic School of Tunisia, La Marsa, Tunisia. Research interests: Cable dynamics, non-linear static and dynamic analysis of cable structures, tensegrity structures, active control of flexible structures.

Maher ABDELGHANI. Assoc. Prof. at the Dept of Mechanical Engineering of the Higher Institute of Applied Sciences and Technologies of Sousse, University of Sousse Tunisia. He obtained his PhD degree from the University of Montpellier II, France, He is a member of the Laboratory of Systems and Applied Mechanics, Polytechnic School of Tunisia, La Marsa, Tunisia. Research interests: structural dynamics, structural health monitoring, system modelling and identification, advanced control theory.

Nabil BEN KAHLA. Currently an Assoc. Prof. at the Dept of Civil Engineering of King Khalid University, Abha, Kingdom of Saudi Arabia. He obtained his PhD degree from the University of Wisconsin-Madison, USA. He is a member of the Laboratory of Systems and Applied Mechanics, Polytechnic School of Tunisia, La Marsa, Tunisia. He is the author of several scientific papers. Research interests: non-linear cable dynamics, guyed masts, tensegrity structures, discrete element methods, active and passive control of flexible structures. 